\title{
ORIGINAL
}

\section{Left ventricular overloading identified by critical care echocardiography is key in weaning-induced pulmonary edema}

\author{
Marine Goudelin ${ }^{1,2}$, Pauline Champy ${ }^{1}$, Jean-Bernard Amiel'1 , Bruno Evrard ${ }^{1,2}$, Anne-Laure Fedou ${ }^{1,2}$, \\ Thomas Daix ${ }^{1,2}$, Bruno François ${ }^{1,2}$ and Philippe Vignon ${ }^{1,2,3,4^{*}}$
}

(c) 2020 Springer-Verlag GmbH Germany, part of Springer Nature

\begin{abstract}
Purpose: To assess the role of left ventricular overload and cumulated fluid balance in the development weaninginduced pulmonary edema (WIPO).

Methods: Ventilated patients in sinus rhythm with COPD and/or heart failure (ejection fraction $\leq 40 \%$ ) were studied. Echocardiography was performed immediately before and during a 30-min spontaneous breathing trial (SBT) using a T-tube. Patients who failed were treated according to echocardiography results before undergoing a second SBT.

Results: Twelve of 59 patients failed SBT, all of them developing WIPO. Patients who succeeded SBT had lower body weight $(-2.5 \mathrm{~kg}[-4.8 ;-1] \mathrm{vs} .+0.75 \mathrm{~kg}[-2.95 ;+5.57]: p=0.02)$ and cumulative fluid balance $(-2326 \mathrm{ml}$ $[-3715 ;+863]$ vs. $+143 \mathrm{ml}[-2654 ;+4434]: p=0.007)$ than those who developed WIPO. SBT-induced central hemodynamic changes were more pronounced in patients who developed WIPO, with higher $E$ wave velocity $(122 \mathrm{~cm} / \mathrm{s}$ [92; 159] vs. $93 \mathrm{~cm} / \mathrm{s}[74 ; 109]: p=0.017)$ and $E / A$ ratio $(2.1[1.2 ; 3.6]$ vs. $0.9[0.8 ; 1.4]: p=0.001)$, and shorter E wave deceleration time (85 ms [72; 125] vs. 147 ms [103; 175]: $p=0.004)$. After echocardiography-guided treatment, all patients who failed the first SBT were successfully extubated. Fluid balance was then negative $(-2224 \mathrm{ml}$ $[-7056 ;+100]$ vs. $+146 \mathrm{ml}[-2654 ;+4434]: p=0.005)$. Left ventricular filling pressures were lower $\left(E / E^{\prime}: 7.3[5 ; 10.4]\right.$ vs. 8.9 [5.9; 13.1$]$ : $p=0.028)$; SBT-induced increase in $E$ wave velocity $(+10.6 \%[-2.7 /+18]$ vs. $+25.6 \%[+12.7 /+49]$ : $p=0.037)$ and of mitral regurgitation area were significantly smaller.

Conclusion: In high-risk patients, WIPO appears related to overloaded left ventricle associated with excessive fluid balance. SBT-induced central hemodynamic changes monitored by CCE help in guiding therapy for successful weaning.

Keywords: Ventilator weaning, Pulmonary edema, Water-electrolyte balance, Echocardiography, Echocardiography Doppler
\end{abstract}

*Correspondence: philippe.vignon@unilim.fr

${ }^{4}$ Réanimation Polyvalente, CHU Dupuytren, 2 Avenue Martin Luther king, 87000 Limoges, France

Full author information is available at the end of the article

\section{Introduction}

Since prolonged invasive mechanical ventilation is associated with increased morbidity and mortality, patients must be weaned from the ventilator as soon as possible [1]. Nevertheless, weaning failure rate ranges between 26 and 42\% [2]. Spontaneous breathing trial (SBT) has been compared to a physical exercise [3]. The transition 
from positive to negative intrathoracic pressure alters unfavorably cardiac loading conditions, the net result being an abrupt increase in left ventricular (LV) filling pressures [4]. Accordingly, weaning pulmonary edema (WIPO) is a leading cause of weaning failure $[5,6]$, especially in high-risk patients with an underlying cardiopathy, chronic obstructive pulmonary disease (COPD), or obesity [7].

Negative passive leg raise reflecting the absence of fluid responsiveness $[7,8]$ and high $\mathrm{B}$-type natriuretic peptide (BNP) [9-12] or NT pro-BNP $[9,13]$ levels have been shown to be associated with an increased risk of weaning failure. In addition, guidance of fluid management based on daily measurement of plasma BNP concentration significantly reduces fluid balance and shortens the duration of mechanical ventilation, especially in the presence of LV failure [14]. Taken together, these results underline the pivotal contribution of cumulated hydric balance and potentially associated volume overload in the development of WIPO [15].

This study aimed at assessing the ability of critical care echocardiography (CCE) [16] to accurately track SBTinduced central hemodynamic changes in patients at high risk of WIPO and its value for guiding therapy after a failed SBT due to WIPO.

\section{Material and methods}

This prospective, observational, single-center study was conducted in the Intensive Care Unit (ICU) of Limoges University Hospital over a 28-month period. The study was approved by the Ethics Committee of the Sociéte de Réanimation de Langue Française which waived informed consent.

\section{Patients}

Patients were eligible if they fulfilled the following inclusion criteria: (1) invasive mechanical ventilation for more than $48 \mathrm{~h}$; (2) medical history of COPD [17] and/ or heart failure with a left ventricular ejection fraction $(\mathrm{LVEF}) \leq 40 \%$; (3) eligibility to perform a weaning trial, as previously described [2] (e-Appendix 1). Patients were not studied if they had at least one of the following criteria: non-sinus rhythm, severe mitral valve disease, mitral valve replacement or repair, tracheostomy, or inadequate imaging quality for Doppler assessment.

In each patient, the following data were recorded on ICU admission: patient's characteristics, reason for ICU admission, medical history, SAPS II [18] and SOFA [19] scores. Data recorded at each weaning trial are detailed in e-Appendix 1. After extubation, patients were followed until ICU discharge to record the need for re-intubation and its reason, and vital status.

\section{Take-home message}

In high-risk patients, weaning-induced pulmonary edema is related to left ventricular overload associated with excessive fluid balance. Critical care echocardiography allows guiding treatment for successful weaning.

\section{Weaning trials}

Weaning trial was considered on a daily basis in a patient under pressure support $[1,2]$. In eligible patients, a 30-min SBT on a T-tube was performed with the oxygen flow set to obtain a $\mathrm{SpO}_{2} \geq 90 \%$ [2]. ST-segment was monitored continuously during SBT to detect potentially induced myocardial ischemia (Intellivue MP 70, Philips Healthcare, Germany).

SBT failure was clinically diagnosed on previously defined criteria [2, 6], and WIPO was confirmed using chest X-ray or lung ultrasound (e-Appendix 1). Patients were then reconnected to the ventilator, and the treatment was modified according to clinical presentation and CCE results [6, 20]: diuretics (LV overload reflected by increased $\mathrm{E}$ wave velocity and/or positive fluid balance), vasodilators including nitrates (hypertension, LV overload, ischemic cardiomyopathy reflected by LV regional wall motion abnormality) and/or angiotensin converting enzyme (ACE) inhibitor (hypertension, LV diastolic dysfunction with elevated filling pressure, worsened mitral regurgitation), or beta-blockers (hypertrophic cardiomyopathy, dynamic obstruction $>30 \mathrm{mmHg}$ measured with continuous wave Doppler) [21]. Elevated LV filling pressure was identified in the presence of grade II or III diastolic dysfunction [22]. Patients were then reassessed daily and underwent a new SBT when weaning criteria were subsequently met. When SBT was clinically successful, the patient was extubated. Extubation failure was defined as the need for re-intubation within $48 \mathrm{~h}$ after extubation [2]. All cases of WIPO were independently confirmed off-line by two intensivists who separately reviewed the medical chart of patients who failed SBT, without having access to CCE data (e-Appendix 1, e-Table 1).

\section{Transthoracic echocardiography}

In all patients, CCE was systematically performed immediately before and at the end of SBT (after $30 \mathrm{~min}$ or earlier at the time of respiratory distress due to failed SBT, before reconnection to the ventilator). This sequence was repeated during subsequent weaning trials, if any. CCE was also systematically performed before re-intubation to determine the cardiac origin of extubation failure, or not. Parameters were 
measured in triplicate and averaged, off-line, on deidentified CCE images, by an independent and experienced investigator who had no access to medical charts (e-Appendix 1).

\section{Statistical analysis}

Considering that WIPO is deemed responsible for approximately $60 \%$ of weaning failures [7] and the large range of weaning failure rate reported in high-risk patients [2], we estimated that 61 patients should be studied to reach $20 \%$ of WIPO-induced SBT failure with a precision of $10 \%$ and an alpha risk of $5 \%$. Quantitative variables were expressed as medians with their 25th and 75 th percentiles and quantitative variables as percentages. No use of previous value or interpolation rule was used in the presence of missing data. Hemodynamic parameters were compared individually between baseline and the first SBT using paired samples Wilcoxon test. Patients were separated into two groups according to the success or failure of SBT, and data were compared between groups using a Mann-Whitney U test or a Chi-squared test. In the subset of patients who failed the first SBT but could be successfully extubated after the second weaning trial, individual parameters recorded at the time of the two SBTs were compared using a paired samples Wilcoxon test. Cutoff values of $E$ wave velocity were identified to diagnose failed SBT due to WIPO, and alternatively to predict successful extubation, with a specificity $>90 \%$. Statistical significance was defined as a $p$ value $<0.05$ (SPSS, Chicago, IL, USA).

\section{Results}

\section{Study population}

Of the 2852 patients admitted to our medical-surgical ICU during the study period, 2364 were mechanically ventilated (83\%) and 361 had a COPD and/or heart failure $(15 \%)$. Of them, $304(84 \%)$ remained under invasive mechanical ventilation for more than $48 \mathrm{~h}$ but 114 died before ventilator weaning, 114 had a non-sinus rhythm, and 17 had exclusion criteria (Fig. 1). Finally, 59 patients were studied (39 men; median age 62 years [52-73]; SAPS2: 38.5 [30-53]; SOFA: 6 [4-9]). Forty-four patients $(75 \%)$ had a documented heart failure, 21 patients $(36 \%)$ had a COPD, 6 of them (10\%) having both (Table 1). No significant ST-segment variations were recorded during SBTs.

\section{First weaning trial}

SBT was first performed after a median duration of mechanical ventilation of $96 \mathrm{~h}$ [54-171]. Fortyseven patients succeeded SBT and were subsequently extubated, whereas the remaining 12 patients $(20 \%)$

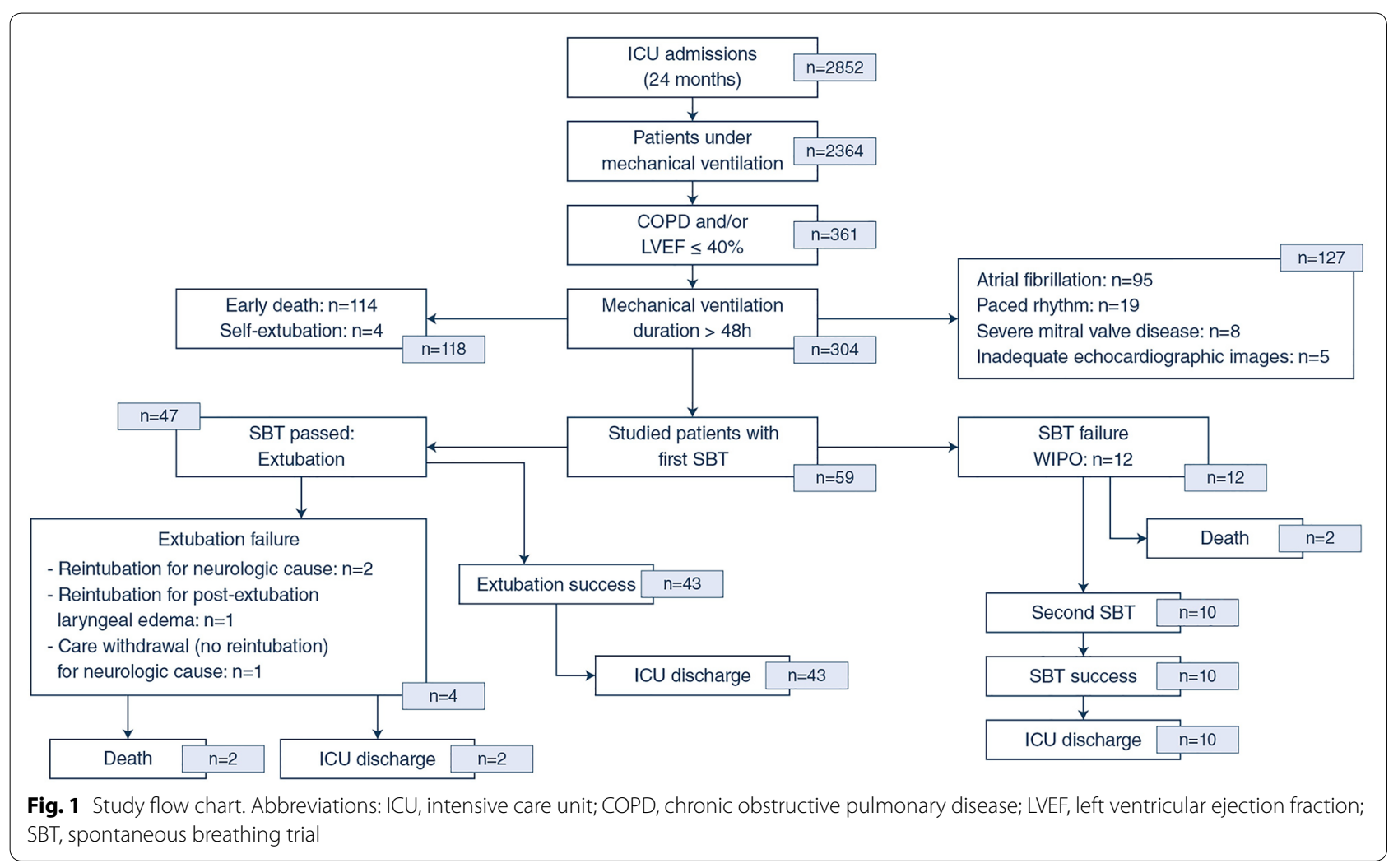


Table 1 Patients characteristics at inclusion according to the result of the first spontaneous breathing trial (SBT)

\begin{tabular}{|c|c|c|c|c|}
\hline & $\begin{array}{l}\text { Study population } \\
(n=59)\end{array}$ & $\begin{array}{l}\text { SBT success } \\
(n=47)\end{array}$ & $\begin{array}{l}\text { SBT failure } \\
(n=12)\end{array}$ & $P$ value \\
\hline Age, years & $62(52-73)$ & $63(52-73)$ & $58(45-70)$ & 0.38 \\
\hline Gender, M/F & $39 / 20$ & $29 / 18$ & $10 / 2$ & 0.157 \\
\hline Heart failure with LVEF $\leq 40 \%(\%)$ & $44(75)$ & $35(74)$ & $9(75)$ & 0.97 \\
\hline COPD (\%) & $20(36)$ & $16(34)$ & $4(33)$ & 0.848 \\
\hline \multicolumn{5}{|l|}{ Reason for ICU admission } \\
\hline Acute pulmonary edema (\%) & $13(22)$ & $10(21)$ & $3(25)$ & 0.78 \\
\hline Cardiogenic shock (\%) & $7(12)$ & $6(13)$ & $1(8)$ & 0.67 \\
\hline Cardiorespiratory arrest (\%) & $7(12)$ & $6(13)$ & $1(8)$ & 0.78 \\
\hline Exacerbation of COPD (\%) & $6(10)$ & $5(11)$ & $1(8)$ & 0.81 \\
\hline Post cardiac surgery (\%) & $9(15)$ & $8(17)$ & $1(8)$ & 0.76 \\
\hline Other (\%) & $17(29)$ & $12(26)$ & $5(41)$ & 0.47 \\
\hline \multicolumn{5}{|l|}{ Medical history } \\
\hline Ischemic cardiomyopathy (\%) & $38(53)$ & $31(66)$ & $7(58)$ & 0.95 \\
\hline Dilated cardiomyopathy (\%) & $11(19)$ & $7(28)$ & $4(33)$ & 0.09 \\
\hline Hypertrophic cardiomyopathy (\%) & $6(10)$ & $6(13)$ & $0(0)$ & 0.22 \\
\hline Severe aortic valve disease (\%) & $0(0)$ & $0(0)$ & $0(0)$ & - \\
\hline Severe pulmonary hypertension (\%) & $0(0)$ & $0(0)$ & $0(0)$ & - \\
\hline Non severe valve disease (\%) & $8(17)$ & $6(13)$ & $2(17)$ & 0.62 \\
\hline Obesity (\%) & $13(22)$ & $10(21)$ & $3(25)$ & 0.64 \\
\hline \multicolumn{5}{|l|}{ Severity scores } \\
\hline SAPS 2 & $38.5(30-53)$ & $39(30-53)$ & $35.5(23.5-51)$ & 0.46 \\
\hline SOFA & $6(4-9)$ & $6(4-9)$ & $6(3-10)$ & 0.72 \\
\hline \multicolumn{5}{|l|}{ Vital status } \\
\hline ICU mortality (\%) & $4(7)$ & $2(4)$ & $2(17)$ & 0.13 \\
\hline \multicolumn{5}{|l|}{ Therapy on inclusion } \\
\hline Bronchodilators (\%) & $9(15)$ & $7(15)$ & $2(16)$ & 0.765 \\
\hline Corticosteroids (\%) & $8(14)$ & $6(13)$ & $2(16)$ & 0.62 \\
\hline Furosemide (\%) & $25(42)$ & $20(43)$ & $5(42)$ & 0.82 \\
\hline Nitrates (\%) & $1(2)$ & $1(2)$ & $0(0)$ & 0.63 \\
\hline Catecholamines (\%) & $17(29)$ & $13(28)$ & $4(33)$ & 0.54 \\
\hline \multicolumn{5}{|l|}{ Respiratory parameters } \\
\hline $\mathrm{FiO}_{2}, \%$ & $30(30-40)$ & $30(30-40)$ & $30(30-37.5)$ & 0.695 \\
\hline Pressure support level, $\mathrm{cm} \mathrm{H}_{2} \mathrm{O}$ & $12(10-14)$ & $12(10-14)$ & $14(11-15)$ & 0.234 \\
\hline PEEP, $\mathrm{cmH}_{2} \mathrm{O}$ & $7(4-10)$ & $6(4-10)$ & $9(7-10)$ & 0.128 \\
\hline $\mathrm{RR}$, breath/min & $19(16-24)$ & $19(16-22)$ & $20(16-20)$ & 0.884 \\
\hline Duration of mechanical ventilation, $\mathrm{h}$ & $110(60-180)$ & $96(54-171)$ & $127(86-241)$ & 0.30 \\
\hline $\mathrm{SaO}_{2}, \%$ & $98(96-99)$ & $100(96-100)$ & 97 (96-99) & 0.59 \\
\hline $\mathrm{PaO}_{2}, \mathrm{mmHg}$ & $142(81-234)$ & $99(85-108)$ & $86(85-120)$ & 0.51 \\
\hline $\mathrm{PaO}_{2} / \mathrm{FiO}_{2}$ & $287(235-351)$ & $287(228-353)$ & $288(241-340)$ & 0.91 \\
\hline $\mathrm{PaCO}_{2}, \mathrm{mmHg}$ & $42(33-60)$ & $36(32-43)$ & $35(32-40)$ & 0.55 \\
\hline \multicolumn{5}{|l|}{ Hydric balance } \\
\hline Weight difference (admission), kg & $-2.0(-4.2 /+1.2)$ & $-2.5(-4.8 /+1)$ & $0.75(-2.95 /+5.6)$ & 0.02 \\
\hline Fluid balance (admission), ml & $-2150(-3505 /+1282)$ & $-2326(-3715 /+863)$ & $146(-2654 /+4434)$ & 0.047 \\
\hline
\end{tabular}

Results are expressed as medians (with 25th and 75th percentiles in parentheses), or as numbers (percentages in parentheses)

COPD chronic obstructive pulmonary disease, SAPS Simplified Acute Physiology score, SOFA sepsis-related organ failure assessment, ICU intensive care unit, PEEP positive end-expiratory pressure, $R R$ respiratory rate 
developed WIPO and were maintained under mechanical ventilation. Four of the 47 extubated patients (8.5\%) developed weaning failure within $48 \mathrm{~h}$ after the planned extubation, but none was of cardiac origin (Fig. 1).

When compared to positive-pressure ventilation, $E$ wave maximal velocity significantly increased $(95 \mathrm{~cm} / \mathrm{s}$ [75-115] vs. $85 \mathrm{~cm} / \mathrm{s}$ [61-100]: $p<0.001)$, E wave deceleration time shortened (128 ms [89-163] vs. $147 \mathrm{~ms}$
[109-206]: $p<0.001), \mathrm{E} / \mathrm{E}$ ' ratio and peak velocity of tricuspid regurgitation increased during SBT (Table 2).

When compared to patients who failed SBT, patients who passed SBT and were extubated had a negative fluid balance during hospitalization $(-2326 \mathrm{ml}[-3715 /+863]$ vs. $+146 \mathrm{ml}[-2654 /+4434]: p=0.047)$ and loss of body weight relative to admission $(-2.5 \mathrm{~kg}[-4.8 /-1.0]$ vs. $+0.75 \mathrm{~kg}[-2.95 /+5.6]: p=0.02)$. No other significant difference was noted between clinical characteristics

Table 2 Hemodynamic parameters and echocardiographic findings obtained immediately before and during the first spontaneous breathing trial (SBT)

\begin{tabular}{|c|c|c|c|c|}
\hline & $\begin{array}{l}\text { General population } \\
(n=59)\end{array}$ & $\begin{array}{l}\text { SBT success } \\
(n=47)\end{array}$ & $\begin{array}{l}\text { SBT failure } \\
(n=12)\end{array}$ & $P$ value \\
\hline \multicolumn{5}{|l|}{ Before SBT } \\
\hline \multicolumn{5}{|c|}{ Hemodynamic parameters } \\
\hline $\mathrm{HR}$, bpm & $94(82-108)$ & $90(80-103)$ & $96(86-113)$ & 0.13 \\
\hline $\mathrm{sBP}, \mathrm{mmHg}$ & $138(130-151)$ & $142(130-153)$ & $131(108-138)$ & 0.08 \\
\hline $\mathrm{dBP}, \mathrm{mmHg}$ & $79(71-87)^{*}$ & $78(71-87)$ & $81(69-88)$ & 0.65 \\
\hline $\mathrm{mBP}, \mathrm{mmHg}$ & $97(87-107)^{*}$ & $98(88-107)^{*}$ & $92(82-110)$ & 0.61 \\
\hline \multicolumn{5}{|c|}{ Echocardiography findings } \\
\hline$E, \mathrm{~cm} / \mathrm{s}$ & $85(61-100)^{*}$ & $83(62-91)$ & $101(84-144)$ & 0.046 \\
\hline$A, \mathrm{~cm} / \mathrm{s}$ & $85(62-109)$ & $86(23-172)$ & $58(37-101)$ & 0.025 \\
\hline$E / A$ & $0.9(0.8-1.4)^{*}$ & $0.9(0.7-1.1)$ & $1.7(1.3-2.4)$ & 0.001 \\
\hline DTE, ms & $147(109-206)^{*}$ & $173(128-213)$ & $107(94-129)$ & 0.002 \\
\hline$E^{\prime}$ lateral, $\mathrm{cm} / \mathrm{s}$ & $9.9(7.5-12.6)$ & $9.9(7.5-12.4)$ & $9.8(7-15.3)$ & 0.66 \\
\hline$E / E^{\prime}$ & $8.4(5.7-11.3)^{*}$ & $9.1(5.6-11)$ & $9.2(6.6-12.5)$ & 0.32 \\
\hline MR/LA area & $0.18(0.14-0.23)$ & $0.2(0.16-0.24)$ & $0.15(0.13-0.31)$ & 0.87 \\
\hline LVEF, \% & $35(24-40)$ & $35(26-40)$ & $24(18-36)$ & 0.083 \\
\hline LVOT VTI, cm & $16.1(12.8-21)$ & $16.6(13.4-20.9)$ & $14.6(8.5-21.2)$ & 0.295 \\
\hline TR peak velocity, $\mathrm{m} / \mathrm{s}$ & $2.96(2.66-3.38)^{*}$ & $2.87(2.53-3.05)$ & $3.39(2.86-3.78)$ & 0.047 \\
\hline \multicolumn{5}{|l|}{ During SBT } \\
\hline \multicolumn{5}{|c|}{ Hemodynamic parameters } \\
\hline $\mathrm{HR}$, bpm & $102(91-115)$ & $96(86-113)$ & $131(113-143)$ & $<0.0001$ \\
\hline $\mathrm{sBP}, \mathrm{mmHg}$ & $146(125-158)$ & $147(125-157)$ & $140(118-168)$ & 0.8 \\
\hline $\mathrm{dBP}, \mathrm{mmHg}$ & $80(70-95)^{*}$ & $80(70-93)$ & $80(65-98)$ & 0.98 \\
\hline $\mathrm{mBP}, \mathrm{mmHg}$ & $104(90-113)^{*}$ & $104(93-113)^{*}$ & $111(72-113)$ & 0.95 \\
\hline \multicolumn{5}{|c|}{ Echocardiography findings } \\
\hline$E, \mathrm{~cm} / \mathrm{s}$ & $95(75-115)^{*}$ & $93(74-109)$ & $122(92-159)$ & 0.017 \\
\hline$A, \mathrm{~cm} / \mathrm{s}$ & $83(68-112)$ & $88(75-114)$ & $60(33-94)$ & 0.012 \\
\hline$E / A$ & $0.9(0.8-1.8)^{*}$ & $0.88(0.8-1.3)$ & $2.1(1.2-3.6)$ & 0.001 \\
\hline DTE, ms & $128(89-163)^{*}$ & $147(103-175)$ & $85(72-125)$ & 0.004 \\
\hline$E^{\prime}$ lateral, $\mathrm{cm} / \mathrm{s}$ & $10.6(8-12.6)$ & $10(8.1-12.3)$ & $11.4(7-16.1)$ & 0.26 \\
\hline$E / E^{\prime}$ & $9.5(7.5-12.7)^{*}$ & $9.6(7.2-12.7)$ & $9.3(7.8-16)$ & 0.84 \\
\hline MR/LA area & $0.21(0.15-0.3)$ & $0.21(0.14-0.24)$ & $0.26(0.17-0.4)$ & 0.57 \\
\hline LVEF, \% & $32(22-42)$ & $33(24-46)$ & $23(13-33)$ & 0.033 \\
\hline LVOT VTI, cm & $16.8(12.8-20.7)$ & $17.5(14.1-20.8)$ & $11.5(7.7-19.2)$ & 0.088 \\
\hline TR peak velocity, $\mathrm{m} / \mathrm{s}$ & $3.18(2.92-3.56)^{*}$ & $3.01(2.73-3.3)$ & $3.67(3.15-4.15)$ & 0.12 \\
\hline
\end{tabular}

$H R$ heart rate, $s B P$ systolic blood pressure, $d B P$ diastolic blood pressure, $m B P$ mean blood pressure, $D T E$ deceleration time of mitral $E$ wave, $M R$ mitral regurgitation, $L A$ left atrium, LVEF left ventricular ejection fraction, LVOT left ventricular outflow tract, VTI velocity-time integral, TR tricuspid regurgitation

*Significant difference between parameters measured under positive pressure ventilation and during the weaning trial 
of both groups, except for a higher heart rate in weaning failure patients (Tables 1 and 2). Under positive pressure ventilation, patients who failed SBT exhibited a higher E wave maximal velocity $(101 \mathrm{~cm} / \mathrm{s}$ [84-144] vs. $83 \mathrm{~cm} / \mathrm{s}$ [62-91]: $p=0.046)$, an increased $E / A$ ratio (1.7 [1.3-2.4] vs. 0.9 [0.7-1.1]: $p=0.001)$, a shorter $E$ wave deceleration time (107 ms [94-129] vs. $173 \mathrm{~ms}$ [128213]: $p=0.002)$ and a higher tricuspid regurgitation peak velocity $(3.39 \mathrm{~m} / \mathrm{s}$ [2.86-3.78] vs. $2.87 \mathrm{~m} / \mathrm{s}$ [2.53-3.05]: $p=0.047)$, while baseline LV ejection fraction tended to be lower (24\% [18-36] vs. $35 \%$ [26-40]: $p=0.083)$. In contrast, no significant difference in the $E$ ' wave maximal velocity and $E / E^{\prime}$ ratio was noted (Table 2 ). During SBT, variations of Doppler parameters were more marked in patients who failed than in those who succeeded the weaning trial: higher increase in $E$ wave maximal velocity $(122 \mathrm{~cm} / \mathrm{s}$ [92-159] vs. $93 \mathrm{~cm} / \mathrm{s}$ [74-109]: $p=0.017)$ and of $E / A$ ratio (2.1 [1.2-3.6] vs. 0.9 [0.8-1.4]: $p=0.001)$, and greater shortening of $E$ wave deceleration time $(85 \mathrm{~ms}$ [72-125] vs. $147 \mathrm{~ms}$ [103-175]: $p=0.004)$. Again, no significant difference was observed for the E' wave maximal velocity and $E / E^{\prime}$ ratio (Table 2 ).

\section{Second weaning trial}

Of the 12 patients who failed the first SBT due to WIPO, two died before subsequent weaning trial. All 10 remaining patients succeeded the second SBT and were successfully extubated without requiring reintubation until ICU discharge (Fig. 1). Median body weight significantly decreased between both SBTs relative to admission $(-5.0 \mathrm{~kg}[-9.5 ;-1.7)]$ vs. $+1.6 \mathrm{~kg}$ $[-2.1 ;+6.5]: p=0.005)$ as did fluid balance $(-2224 \mathrm{ml}$ $[-7056 ;+100)]$ vs. $+146 \mathrm{ml}[-2654 ;+4434]: p=0.005)$.

After the initial SBT failure, 9 of 10 patients received diuretics, 4 patients received ACE inhibitors, one patient received nitrates, and a single patient received betablocker for LV outflow tract dynamic obstruction associated with massive eccentric acute mitral regurgitation secondary to a systolic anterior motion of the mitral valve. Between both weaning trials, median duration of invasive mechanical ventilation reached $59 \mathrm{~h}$ [25.5-103]. Before the second SBT, E wave maximal velocity and $E / E$ ' ratio were significantly lower than before the first SBT $(84 \mathrm{~cm} / \mathrm{s}$ [67-127] vs. $102 \mathrm{~cm} / \mathrm{s}[85-149]: p=0.017$ and $7.3[5.0-10.4]$ vs. $8.9[5.9-13.1]: p=0.028$, respectively). During the second SBT, maximal $E$ wave velocity, $E / A$ and $E / E^{\prime}$ ratios were lower than during the first weaning trial $(100 \mathrm{~cm} / \mathrm{s}$ [85-122] vs. $122 \mathrm{~cm} / \mathrm{s}$ [93-168]: $p=0.009 ; 1.5[0.9-2.3]$ vs. $2.5[1.1-3.6]: p=0.009$; and 7.7 [6.2-9.9] vs. 9.3 [7.5-12.6]: $p=0.047$, respectively), and tricuspid regurgitation peak velocity tended to be lower (2.90 [2.69-3.37] vs. 3.67 [3.00-4.18]: $p=0.068)$ (Table 3). In addition, functional mitral regurgitation jet area normalized by left atrial area significantly decreased during the second SBT $(0.11$ [0.06-0.29] vs. 0.26 [0.200.39 ]; $p=0.028$ ) (Fig. 2). Beta-blocker dramatically reduced both the LV outflow tract obstruction and mitral regurgitation in the patient with hypertrophic cardiomyopathy. Finally, SBT-induced variation of E wave velocity significantly decreased during the second weaning attempt when compared to the initial failed SBT $(+10.6 \%$ $[-2.7 /+18.0]$ vs. $+25.6 \%[+12.7 /+49.0]: p=0.037)$ and was similar to that observed in patients who successfully passed the first weaning trial $(+13.7 \%[-6.4 /+52.1])$. Similar results were obtained for $E / E$, the difference being not statistically significant (Fig. 3 and e-Table 2). Other Doppler indices were not significantly altered by SBT.

\section{Proposed cutoff values}

$E$ velocity $<0.6 \mathrm{~m} / \mathrm{s}$ both at baseline and during SBT had a $100 \%$ specificity and a $15 \%$ sensitivity to predict passed SBT. $E$ velocity $\geq 0.6 \mathrm{~m} / \mathrm{s}$ at baseline and $E \geq 1.2 \mathrm{~m} / \mathrm{s}$ during SBT had a $91 \%$ specificity and $67 \%$ sensitivity to identify failed SBT due to WIPO.

\section{Discussion}

We showed in the present study that high-risk patients who failed SBT due to WIPO exhibited echocardiographic findings consistent with LV overload (i.e., increased preload and afterload) together with excessive cumulated fluid balance. Worsened or acutely developed mitral regurgitation appeared as a contributing factor to SBT failure. CCE-guided therapy helped attending physicians to successfully extubate patients who previously failed SBT due to WIPO, after a mean of 2.5 days.

The 27\% SBT failure rate observed in the present study is in keeping with that commonly reported in a high-risk population [2]. As previously reported [23], our patients who developed WIPO had significantly higher maximal $E$ wave velocity and $E / A$ ratio, shorter $E$ wave deceleration time, lower LVEF, and higher tricuspid regurgitation peak velocity under positive-pressure ventilation, when compared to their counterparts. This presumably reflects the incapacity of their cardiovascular system to sustain SBT-induced abrupt increase in cardiac work load [3-6]. LV was abruptly overloaded by SBT as reflected by a significant rise of maximal $E$ wave velocity and $E / A$ ratio, a significant shortening of $E$ wave deceleration time [23, 24], and augmented tricuspid regurgitation peak velocity used as a surrogate of systolic pulmonary artery pressure [25]. Not surprisingly, these changes were even more pronounced in the subset of our patients who failed SBT due to WIPO. $E / E^{\prime}$, a routinely used indicator of LV filling pressure, was in the lower range at baseline presumably because a large proportion of our high-risk patients received diuretics before 
Table 3 Hemodynamic parameters and echocardiography findings in the subset of patients who failed the first SBT but succeeded the second weaning trial

\begin{tabular}{|c|c|c|c|}
\hline & $\begin{array}{l}\text { 1st failed SBT } \\
(n=10)\end{array}$ & $\begin{array}{l}\text { 2nd passed SBT } \\
(n=10)\end{array}$ & $P$ value \\
\hline \multicolumn{4}{|l|}{ Before weaning trials } \\
\hline \multicolumn{4}{|l|}{ Hemodynamic parameters } \\
\hline $\mathrm{HR}, \mathrm{bpm}$ & $107(92-126)^{*}$ & $102(91-114)$ & 0.308 \\
\hline $\mathrm{sBP}, \mathrm{mmHg}$ & $131(108-142)$ & $144(120-160)$ & 0.475 \\
\hline $\mathrm{dBP}, \mathrm{mmHg}$ & $82(68-90)$ & $82(77-91)$ & 0.477 \\
\hline $\mathrm{mBP}, \mathrm{mmHg}$ & $92(82-111)$ & $102(90-116)$ & 0.312 \\
\hline \multicolumn{4}{|l|}{ Echocardiography parameters } \\
\hline$E, \mathrm{~cm} / \mathrm{s}$ & $102(85-149)$ & $84(67-127)$ & 0.017 \\
\hline$A, \mathrm{~cm} / \mathrm{s}$ & $58(41-110)$ & $62(48-93)$ & 0.88 \\
\hline$E / A$ & $1.7(1.2-2.7)$ & $1.3(0.8-2.1)$ & 0.139 \\
\hline DTE, ms & $103(92-134)$ & $133(103-184)$ & 0.139 \\
\hline$E^{\prime}$ lateral, $\mathrm{cm} / \mathrm{s}$ & $11.1(7.6-16.4)$ & $13.5(8.8-15.4)$ & 0.646 \\
\hline$E / E^{\prime}$ & $8.9(5.9-13.1)$ & $7.3(5-10.4)$ & 0.028 \\
\hline MR/LA area & $0.15(0.14-0.2)$ & $0.11(0.06-0.17)$ & 0.249 \\
\hline LVEF, \% & $30(20-36)$ & $34(27-38)$ & 0.161 \\
\hline LVOT VTI, cm & $14.6(9.3-22.6)$ & $15.0(10.9-19)$ & 0.678 \\
\hline TR peak velocity, $\mathrm{m} / \mathrm{s}$ & $3.43(2.69-4.52)$ & $3.2(2.64-3.48)$ & 0.285 \\
\hline \multicolumn{4}{|l|}{ During weaning trials } \\
\hline \multicolumn{4}{|l|}{ Hemodynamic parameters } \\
\hline $\mathrm{HR}$, bpm & $131(112-138)$ & $108(99-111)$ & 0.011 \\
\hline $\mathrm{sBP}, \mathrm{mmHg}$ & $143(125-168)$ & $133(113-156)$ & 0.919 \\
\hline $\mathrm{dBP}, \mathrm{mmHg}$ & $88(63-99)$ & $77(70-78)$ & 0.308 \\
\hline $\mathrm{mBP}, \mathrm{mmHg}$ & $112(72-115)$ & $94(85-106)$ & 0.507 \\
\hline \multicolumn{4}{|l|}{ Echocardiography parameters } \\
\hline$E, \mathrm{~cm} / \mathrm{s}$ & $122(93-168)$ & $100(85-122)$ & 0.009 \\
\hline$A, \mathrm{~cm} / \mathrm{s}$ & $60(34-103)$ & $67(48-93)$ & 0.48 \\
\hline$E / A$ & $2.5(1.1-3.6)$ & $1.5(0.9-2.3)$ & 0.009 \\
\hline DTE, ms & $81(71-128)$ & $130(97-133)$ & 0.093 \\
\hline$E^{\prime}$ lateral, $\mathrm{cm} / \mathrm{s}$ & $12.5(8.9-16.4)$ & $14.2(8.5-15.6)$ & 0.878 \\
\hline$E / E^{\prime}$ & $9.3(7.5-12.6)$ & $7.7(6.2-9.9)$ & 0.047 \\
\hline MR/LA area & $0.26(0.2-0.39)$ & $0.11(0.06-0.29)$ & 0.028 \\
\hline LVEF, \% & $26(16-35)$ & $29(26-36)$ & 0.401 \\
\hline LVOT VTI, cm & $14(8.9-20.1)$ & $15.5(12.6-20)$ & 0.263 \\
\hline TR peak velocity, $\mathrm{m} / \mathrm{s}$ & $3.67(3-4.18)$ & $2.90(2.69-3.37)$ & 0.068 \\
\hline \multicolumn{4}{|l|}{ Hydric balance } \\
\hline Weight difference (admission), kg & $+1.6(-2.1 /+6.5)$ & $-5.0(-9.5 /-1.7)$ & 0.005 \\
\hline Fluid balance (admission), ml & $+146(-2654 /+4434)$ & $-2224(-7056 /+100)$ & 0.005 \\
\hline
\end{tabular}

$H R$ heart rate, $s B P$ systolic blood pressure, $A B P$ diastolic blood pressure, $m B P$ mean blood pressure, $D T E$ deceleration time of mitral $E$ wave, $M R$ mitral regurgitation, $L A$ left atrium, LVEF left ventricular ejection fraction, LVOT left ventricular outflow tract, VTI velocity-time integral, $T R$ tricuspid regurgitation

*Significant difference between parameters measured under positive pressure ventilation and during the weaning trial

SBT (median hydric balance: $+146 \mathrm{~mL}$ ). In addition, $E / E^{\prime}$ failed to significantly increase during SBT because $E^{\prime}$ also increased presumably in response to sympathetic stimulation [26]. Maximal $E$ wave velocity-which directly reflects the pressure gradient between the left atrium and the LV at early diastole-is the result of both LV loading conditions and relaxation properties [27]. Worsened functional mitral regurgitation during SBT may contribute to increase $\mathrm{E}$ wave velocity and subsequent weaning failure in response to augmented LV preload and afterload [13]. The increase in both $\mathrm{E}$ wave maximal velocity and $\mathrm{E} / \mathrm{A}$ ratio associated with the shortening of $\mathrm{E}$ wave 


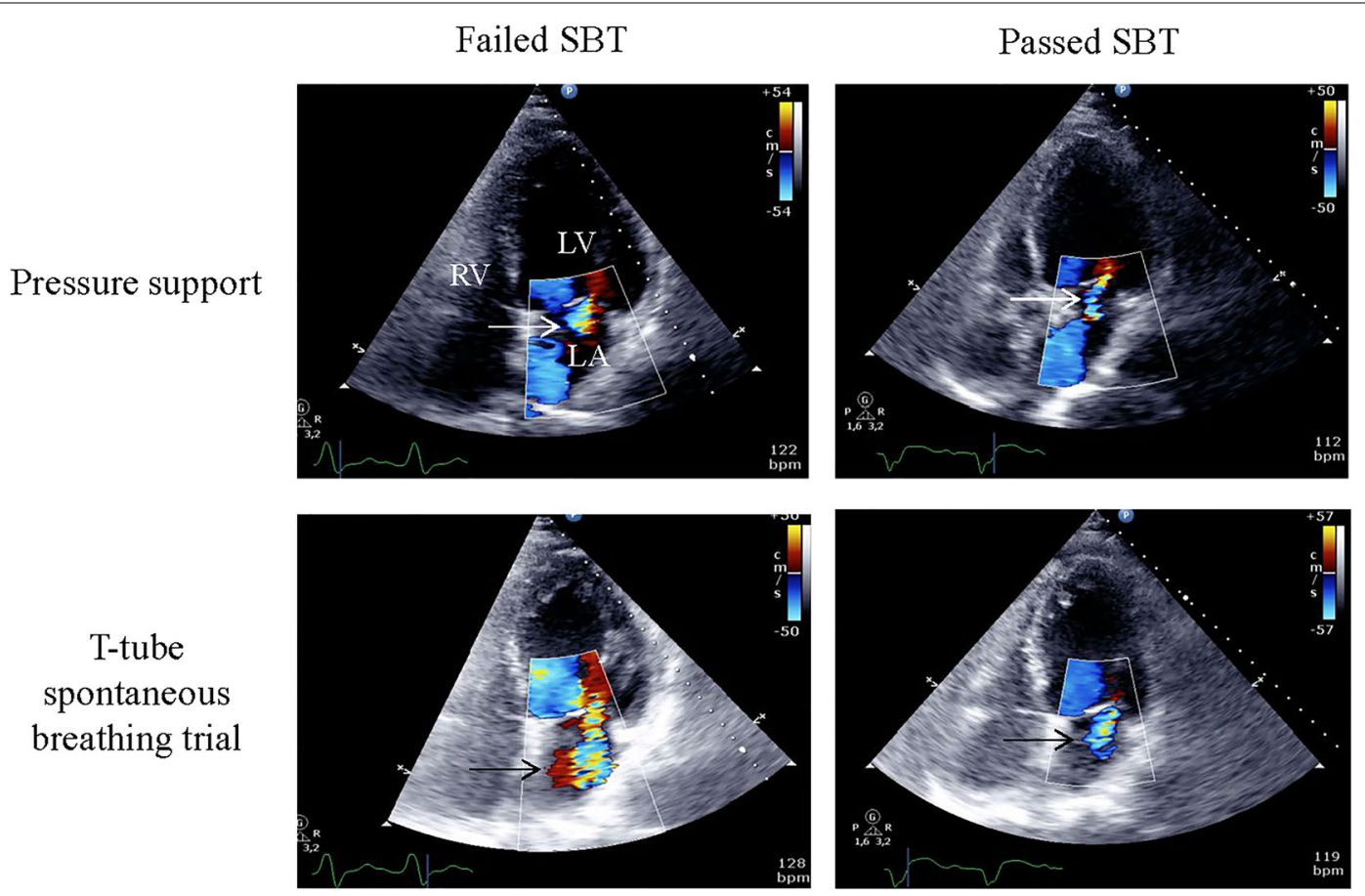

Fig. 2 Variations of functional mitral regurgitation severity during spontaneous breathing trials (SBT) and effect of echocardiography-guided therapy. Central functional mitral regurgitation at baseline (upper left, arrow) significantly increased during the failed spontaneous breathing trial (lower left panel, arrow). After a treatment associating diuretics and angiotensin converting enzyme inhibitors, the central mitral regurgitation which remained trivial at baseline (upper right panel, arrow) failed to significantly worsen during the second weaning trial (lower right panel, arrow), and the patient was successfully extubated

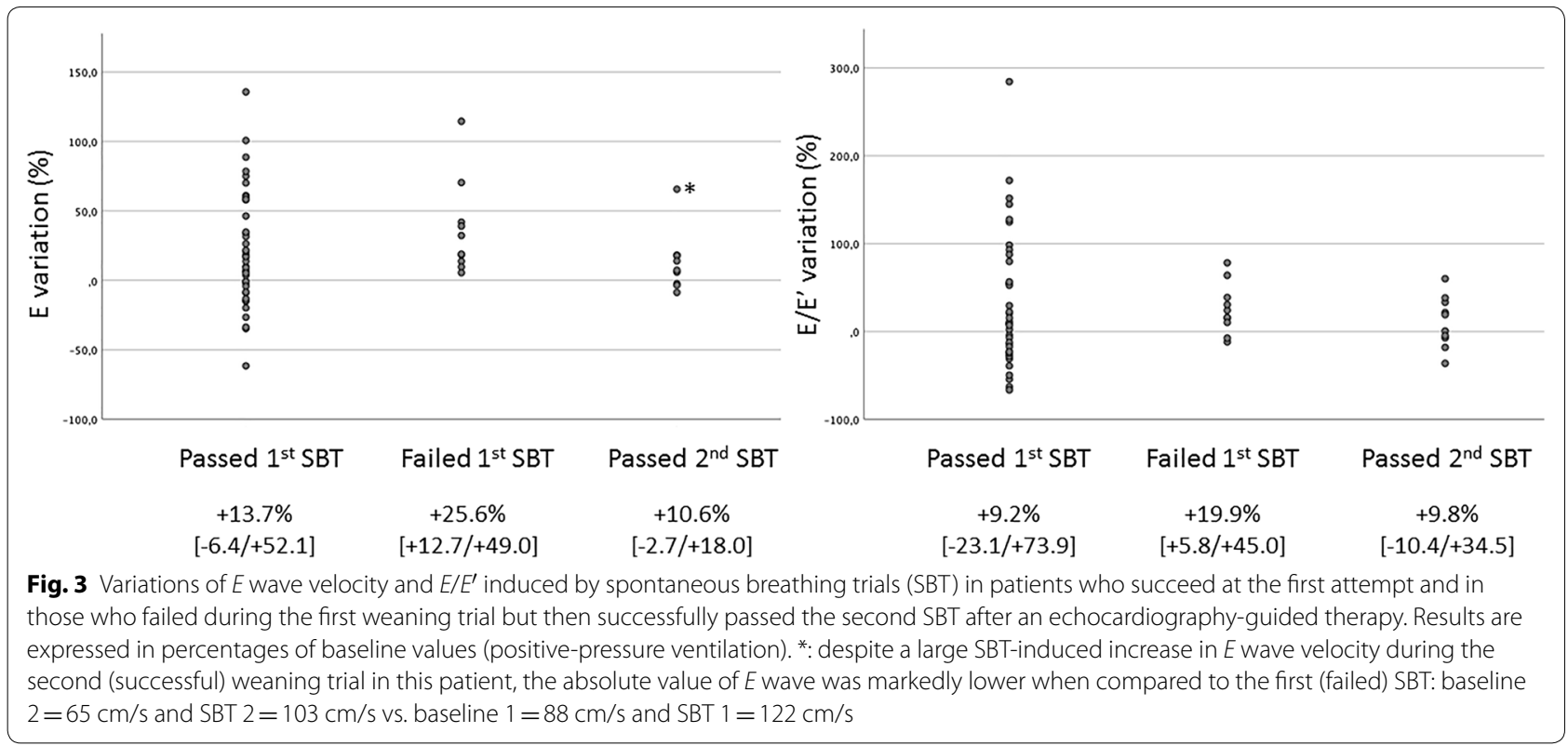

deceleration time concordantly reflect a significant rise of LV filling pressure [4], since these parameters are correlated to the pulmonary capillary pressure in patients with
LV systolic dysfunction [22, 27, 28], a large proportion of our study population. In light of the absence of significant ST-segment variations during SBTs, this suggests that 
the abrupt rise of LV filling pressure induced by SBT was related to LV overload rather than deteriorated LV relaxation or compliance of ischemic origin $[4,6]$. This pathophysiologic mechanism is also supported by the higher weight gain and cumulative fluid balance relative to ICU admission in patients who failed SBT [8-13]. Of note, with the exception of higher heart rate-a rather nonspecific sign-no clinical parameter was significantly associated with SBT failure in our patients. This underlines the additional value of monitoring central hemodynamics using CCE during weaning trial in patients at high risk of WIPO [15, 29, 30].

In our high-risk patients who passed SBT, the absence of relevant central hemodynamic changes identified with CCE during the weaning trial confirmed the feasibility of extubation. This diagnostic strategy was successful since no extubation failure of cardiac origin was observed after tracheal tube withdrawal. In those who failed the first SBT, CCE was used to guide therapy according to a predefined therapeutic algorithm. Since a relevant increase in $\mathrm{E}$ wave velocity consistent with an overloaded LV was observed in most patients at the time of SBT failure, diuretics and vasodilators were predominantly used to reduce both LV preload and afterload [31,32]. A beta-blocker was used in a patient with a dynamic LV outflow tract obstruction and severe acute mitral regurgitation related to a systolic anterior motion of the mitral valve [33]. After these therapeutic changes, all patients who initially failed the first SBT could be subsequently extubated successfully. Immediately before and during passed second $\mathrm{SBT}$, maximal $\mathrm{E}$ wave velocity, $E / A$ and $E / E^{\prime}$ ratios, and tricuspid regurgitation peak velocity were lower than at the time of failed first weaning trial, whereas $E$ wave deceleration time was prolonged. In our patients with relevant functional mitral insufficiency at baseline, the regurgitant volume reflected by the jet area significantly decreased between the two weaning trials. The resulting relative increase in $\mathrm{E}$ wave velocity induced by the weaning trial was significantly lower during the second (passed) SBT when compared to the first (failed) one. These changes suggest that CCE-guided therapy unloaded LV within a median delay of 2.5 days. The significant decrease in body weight and cumulated fluid balance relative to the first weaning trial suggest a link between LV overload and excessive fluid balance at the origin of WIPO. Volume overload has been associated with worse weaning outcome $[34,35]$.

The present results may not be reproducible using other types of weaning trial. Low-pressure support level without PEEP has been shown to induce less central hemodynamic variations than SBT using a T-piece [36]. In a recent meta-analysis, Sklar et al. [37] suggested that SBT using a T-piece best mimics changes in cardiac and respiratory loading conditions resulting from extubation. In our high-risk population, its use has presumably sensitized SBT to reveal WIPO. Since the precise cutoff value of LV filling pressure which would precipitate the development of WIPO is unknown for a given patient [4] and most Doppler indices are in the intermediate range at baseline $[38,39]$, the magnitude of SBT-induced variations should be precisely determined and interpreted in lights of clinical presentation, the patient being his own control. When compared to right-heart catheterization which is considered the reference for the diagnosis of WIPO [4], CCE is less invasive, rapid to implement, easy to use, and depicts certain mechanisms of WIPO which are not accessible to conventional monitoring systems, such as LV outflow tract obstruction as in one of our patient. In this specific clinical setting, right heart catheterization typically depicts elevated pulmonary artery occlusion pressure and low cardiac index which may erroneously lead to diuretics, vasodilators or even inotropes therapy, all of them exacerbating the hemodynamic burden [33].

This prospective study is limited by its strict eligibility criteria, excluding patients with non-sinus rhythm and selecting high-risk patients as reflected by the $100 \%$ prevalence of WIPO as a cause of SBT failure. Accordingly, the efficacy of CCE-based strategy of weaning needs to be confirmed in a broader population [40], using the herein proposed E wave cutoff values to guide volume depletion and vasodilators administration. The diagnosis of WIPO relied on both the clinical presentation and CCE findings, as advocated [6], rather than on invasive measurement of pulmonary artery occlusion pressure [4], and lung ultrasound was not systematically used. Nevertheless, this study is the first to establish a link between SBT-induced central hemodynamic changes, overloaded LV, positive fluid balance, and WIPO.

\section{Conclusion}

SBT failure due to WIPO appears related to overloaded $\mathrm{LV}$, including the worsening or development of mitral regurgitation, associated with excessive cumulated fluid balance. SBT-induced central hemodynamic changes monitored by CCE helps guiding weaning of patients at high risk of WIPO.

\section{Electronic supplementary material}

The online version of this article (https://doi.org/10.1007/s00134-020-06061-y) contains supplementary material, which is available to authorized users.

\section{Author details}

${ }^{1}$ Medical-Surgical Intensive Care Unit, Dupuytren Teaching Hospital, 87000-Limoges, France. ${ }^{2}$ Inserm CIC 1435, Dupuytren Teaching Hospital, 87000-Limoges, France. ${ }^{3}$ Faculty of Medicine, University of Limoges, 87000-Limoges, France. ${ }^{4}$ Réanimation Polyvalente, CHU Dupuytren, 2 Avenue Martin Luther king, 87000 Limoges, France. 


\section{Author contributions:}

MG and PV included patients, analyzed the data and drafted the manuscript. $P C, J B A, B E, A L F, T D$ and $B F$ included patients and critically reviewed the manuscript. All authors read and approved the final version of the manuscript.

\section{Funding}

None.

\section{Compliance with ethical standards}

\section{Conflict of interest}

Authors has no conflict of interest to declare with respect to this work.

\section{Publisher's Note}

Springer Nature remains neutral with regard to jurisdictional claims in published maps and institutional affiliations.

Received: 12 December 2019 Accepted: 17 April 2020 Published online: 6 May 2020

\section{References}

1. Girard TD, Alhazzani W, Kress JP, Ouellette DR, Schmidt GA, Truwit JD et al (2017) An Official American Thoracic Society/American College of Chest Physicians Clinical Practice Guideline: Liberation from Mechanical Ventilation in Critically III Adults. Rehabilitation Protocols, Ventilator Liberation Protocols, and Cuff Leak Tests. Am J Respir Crit Care Med 195:120-133

2. Boles J-M, Bion J, Connors A, Herridge M, Marsh B, Melot C, Pearl R, Silverman H, Stanchina M, Vieillard-Baron A, Welte T (2007) Weaning from mechanical ventilation. Eur Respir J 29:1033-1056

3. Pinsky MR (2000) Breathing as exercise: the cardiovascular response to weaning from mechanical ventilation. Intensive Care Med 26:1164-1166

4. Lemaire F, Teboul JL, Cinotti L, Giotto G, Abrouk F, Steg G, Macquin-Mavier I, Zapol WM (1988) Acute left ventricular dysfunction during unsuccessful weaning from mechanical ventilation. Anesthesiology 69:171-179

5. Perren A, Brochard L (2013) Managing the apparent and hidden difficulties of weaning from mechanical ventilation. Intensive Care Med 39:1885-1895

6. Teboul J-L, Monnet X, Richard C (2010) Weaning failure of cardiac origin: recent advances. Crit Care 14:211

7. Liu J, Shen F, Teboul J-L, Anguel N, Beurton A, Bezaz N, Richard C, Monnet $X$ (2016) Cardiac dysfunction induced by weaning from mechanical ventilation: incidence, risk factors, and effects of fluid removal. Crit Care 20:369

8. Dres M, Teboul J-L, Anguel N, Guerin L, Richard C, Monnet X (2015) Passive leg raising performed before a spontaneous breathing trial predicts weaning-induced cardiac dysfunction. Intensive Care Med 41:487-494

9. Zapata L, Vera P, Roglan A, Gich I, Ordonez-Llanos J, Betbesé AJ (2011) B-type natriuretic peptides for prediction and diagnosis of weaning failure from cardiac origin. Intensive Care Med 37:477-485

10. Mekontso-Dessap A, de Prost N, Girou E, Braconnier F, Lemaire F, BrunBuisson C, Brochard L (2006) B-type natriuretic peptide and weaning from mechanical ventilation. Intensive Care Med 32:1529-1536

11. Chien J-Y, Lin M-S, Huang Y-CT, Chien Y-F, Yu C-J, Yang P-C (2008) Changes in B-type natriuretic peptide improve weaning outcome predicted by spontaneous breathing trial. Crit Care Med 36:1421-1426

12. Dres M, Teboul J-L, Anguel N, Guerin L, Richard C, Monnet X (2014) Extravascular lung water, B-type natriuretic peptide, and blood volume contraction enable diagnosis of weaning-induced pulmonary edema. Crit Care Med 42:1882-1889

13. Gerbaud E, Erickson M, Grenouillet-Delacre M, Beauvieux MC, Coste P, Durrieu-Jaïs C, Hilbert G, Castaing Y, Vargas F (2012) Echocardiographic evaluation and $\mathrm{N}$-terminal pro-brain natriuretic peptide measurement of patients hospitalized for heart failure during weaning from mechanical ventilation. Minerva Anestesiol 78:415-425

14. Mekontso Dessap A, Roche-Campo F, Kouatchet A, Tomicic V, Beduneau G, Sonneville R, Cabello B, Jaber S, Azoulay E, Castanares-Zapatero D, Devaquet J, Lellouche F, Katsahian S, Brochard L (2012) Natriuretic peptide-driven fluid management during ventilator weaning: a randomized controlled trial. Am J Respir Crit Care Med 186:1256-1263

15. Vignon P (2018) Cardiovascular failure and weaning. Ann Transl Med 6:354

16. Mayo PH, Beaulieu Y, Doelken P, Feller-Kopman D, Harrod C, Kaplan A Oropello J, Vieillard-Baron A, Axler O, Lichtenstein D, Maury E, Slama M, Vignon P (2009) American College of Chest Physicians/La Société de Réanimation de Langue Française statement on competence in critical care ultrasonography. Chest 135:1050-1060

17. Vestbo J, Hurd SS, Agustí AG, Jones PW, Vogelmeier C, Anzueto A, Barnes PJ, Fabbri LM, Martinez FJ, Nishimura M, Stockley RA, Sin DD, RodriguezRoisin R (2013) Global strategy for the diagnosis, management, and prevention of chronic obstructive pulmonary disease: GOLD executive summary. Am J Respir Crit Care Med 187:347-365

18. Le Gall JR, Lemeshow S, Saulnier F (1993) A new Simplified Acute Physiology Score (SAPS II) based on a European/North American multicenter study. JAMA 270:2957-2963

19. Vincent JL, Moreno R, Takala J, Willatts S, De Mendonça A, Bruining H, Reinhart CK, Suter PM, Thijs LG (1996) The SOFA (Sepsis-related Organ Failure Assessment) score to describe organ dysfunction/failure. On behalf of the Working Group on Sepsis-Related Problems of the European Society of Intensive Care Medicine. Intensive Care Med 22:707-710

20. Routsi C, Stanopoulos I, Kokkoris S, Sideris A, Zakynthinos S (2019) Weaning failure of cardiovascular origin: how to suspect, detect and treat-a review of the literature. Ann Intensive Care 9:6

21. Maron MS, Olivotto I, Zenovich AG, Link MS, Pandian NG, Kuvin JT, Nistri S, Cecchi F, Udelson JE, Maron BJ (2006) Hypertrophic cardiomyopathy is predominantly a disease of left ventricular outflow tract obstruction. Circulation 114:2232-2239

22. Nagueh SF, Smiseth OA, Appleton CP, Byrd BF 3rd, Dokainish H, Edvardsen T, Flachskampf FA, Gillebert TC, Klein AL, Lancellotti P, Marino P, Oh JK, Alexandru Popescu B, Waggoner AD (2016) Recommendations for the Evaluation of Left Ventricular Diastolic Function by Echocardiography: An Update from the American Society of Echocardiography and the European Association of Cardiovascular Imaging. Eur Heart J Cardiovasc Imaging 17:1321-1360

23. Caille V, Amiel J-B, Charron C, Belliard G, Vieillard-Baron A, Vignon P (2010) Echocardiography: a help in the weaning process. Crit Care 14:R120

24. Ait-Oufella H, Tharaux P-L, Baudel J-L, Vandermeersch S, Meyer P, Tonnellier M, Dussaule JC, Guidet B, Offenstadt G, Maury E (2007) Variation in natriuretic peptides and mitral flow indexes during successful ventilatory weaning: a preliminary study. Intensive Care Med 33:1183-1186

25. Vignon P (2019) Assessment of pulmonary arterial pressure using critical care echocardiography: dealing with the Yin and the Yang? Crit Care Med 47:126-128

26. Nagueh SF, Sun H, Kopelen HA, Middleton KJ, Khoury DS (2001) Hemodynamic determinants of the mitral annulus diastolic velocities by tissue Doppler. J Am Coll Cardiol 37:278-285

27. Appleton CP, Hatle LK, Popp RL (1988) Relation of transmitral flow velocity patterns to left ventricular diastolic function: new insights from a combined hemodynamic and Doppler echocardiographic study. J Am Coll Cardiol 12:426-440

28. Vanoverschelde JL, Raphael DA, Robert AR, Cosyns JR (1990) Left ventricular filling in dilated cardiomyopathy: relation to functional class and hemodynamics. J Am Coll Cardiol 15:1288-1295

29. Vignon P, Repessé X, Vieillard-Baron A, Maury E (2016) Critical care ultrasonography in acute respiratory failure. Crit Care 20:228

30. Mayo P, Volpicelli G, Lerolle N, Schreiber A, Doelken P, Vieillard-Baron A (2016) Ultrasonography evaluation during the weaning process: the heart, the diaphragm, the pleura and the lung. Intensive Care Med 42:1107-1117

31. Voors AA, de Jong RM (2008) Treating diastolic heart failure. Heart Br Card Soc 94:971-972

32. Elkayam U (1996) Nitrates in the treatment of congestive heart failure. Am J Cardiol 77:41C-51C

33. Adamopoulos C, Tsagourias M, Arvaniti K, Veroniki F, Matamis D (2005) Weaning failure from mechanical ventilation due to hypertrophic obstructive cardiomyopathy. Intensive Care Med 31:734-737

34. Frutos-Vivar F, Ferguson ND, Esteban A, Epstein SK, Arabi Y, Apezteguía C, González M, Hill NS, Nava S, D’Empaire G, Anzueto A (2006) Risk factors 
for extubation failure in patients following a successful spontaneous breathing trial. Chest 130:1664-1671

35. National Heart, Lung, and Blood Institute Acute Respiratory Distress Syndrome (ARDS)Clinical Trials Network, Wiedemann HP, Wheeler AP, Bernard GR, Thompson BT, Hayden D, deBoisblanc B, Connors AF Jr, Hite RD, Harabin AL (2006) Comparison of two fluid-management strategies in acute lung injury. N Engl J Med 354:2564-2575

36. Cabello B, Thille AW, Roche-Campo F, Brochard L, Gómez FJ, Mancebo J (2010) Physiological comparison of three spontaneous breathing trials in difficult-to-wean patients. Intensive Care Med 36:1171-1179

37. Sklar MC, Burns K, Rittayamai N, Lanys A, Rauseo M, Chen L, Dres M, Chen GQ, Goligher EC, Adhikari NKJ, Brochard L, Friedrich JO (2017) Effort to Breathe with Various Spontaneous Breathing Trial Techniques. A Physiologic Meta-analysis. Am J Respir Crit Care Med 195:1477-1485
38. Moschietto S, Doyen D, Grech L, Dellamonica J, Hyvernat H, Bernardin G (2012) Transthoracic Echocardiography with Doppler Tissue Imaging predicts weaning failure from mechanical ventilation: evolution of the left ventricle relaxation rate during a spontaneous breathing trial is the key factor in weaning outcome. Crit Care 16:R81

39. Lamia B, Maizel J, Ochagavia A, Chemla D, Osman D, Richard C, Teboul JL (2009) Echocardiographic diagnosis of pulmonary artery occlusion pressure elevation during weaning from mechanical ventilation. Crit Care Med 37:1696-1701

40. Al-Omari MA, Finstuen J, Appleton CP, Barnes ME, Tsang TSM (2008) Echocardiographic assessment of left ventricular diastolic function and filling pressure in atrial fibrillation. Am J Cardiol 101:1759-1765 